\title{
Oscillation criteria for an advanced 2-D discrete system
}

\author{
Chunhua Yuan ${ }^{\mathrm{a}}$, Liang Zhang ${ }^{\mathrm{b}}$ and Jian $\mathrm{Liu}^{\mathrm{c}}$
}

School of Mathematical Science, University of Jinan, Jinan, 250022, China

ayuanchunhua72@163.com, ${ }^{\mathrm{b}} 522599239 @ q q . c o m,{ }^{\mathrm{C}}$ liujian1990@163.com

Keywords: 2-D discrete system, Characteristic equation, Oscillation.

Abstract. This paper is concerned with oscillatory behavior of an advanced 2-D discrete system. Some necessary and sufficient conditions for the oscillation of all solutions of the advanced 2-D discrete system are established. Numerical examples are provided to demonstrate the main results of this paper.

\section{Introduction}

2-D discrete systems arise in applications involving image processing, random walk problems, population dynamics with spatial migrations and finite difference schemes[1-4]. In recent years, lots of results for oscillatory behavior of 2-D discrete systems have been obtained (see [3-11] and the references therein).

In this paper, we are concerned with the oscillatory behavior of the advanced 2-D discrete system:

$$
u_{m+1, n}+u_{m, n+1}-p u_{m, n}+q u_{m+k, n+l}=0, \quad n, m=0,1,2, \ldots
$$

where $p, q$ are real numbers, $k, l$ are nonnegative integers with $k^{2}+l^{2} \neq 0$. However, to the best of our knowledge, no research has been done on the oscillatory behavior of the advanced 2-D discrete system (1). In this paper, we establish some necessary and sufficient conditions for the oscillation of all solutions of the advanced 2-D discrete system (1).

Eq. 1 is said to be an advanced 2-D discrete system if $k$, l are nonnegative integers with $k^{2}+l^{2} \neq 0$.

A solution of Eq. 1 is a real double sequence $\left\{u_{m, n}\right\}$ which is defined for $m \geq 0, n \geq 0$ and satisfies Eq. 1 for $m \geq 0, n \geq 0$.

A solution $\left\{u_{m, n}\right\}$ of Eq. 1 is said to be eventually positive (or negative) if $u_{m, n}>0$ (or $u_{m, n}<0$ ) for large numbers $m \geq 0$ and $n \geq 0$. It is said to be oscillatory if it is neither eventually positive nor eventually negative. Eq. 1 is called oscillatory if all of its nontrivial solutions are oscillatory.

\section{Preliminary}

On basis of Corollary 2.9 in [4], we can easily obtain the following lemma, which is used in the proofs of the main results in the next section.

Lemma 1 The following statements are equivalent:

1) Every solution of Eq. 1 is oscillatory.

2) The characteristic equation $\lambda+\mu-p+q \lambda^{k} \mu^{l}=0$ of Eq. 1 has no positive roots.

\section{Main Results}

Theorem 1. Assume that $k>0$ and $l>0$. Then every solution of Eq. 1 oscillates if and only if $p \leq 0$ and $q \geq 0$.

Proof. When $k>0$ and $l>0$, then the characteristic equation of Eq. 1 is

$$
\phi(p, q, \lambda, \mu)=\lambda+\mu-p+q \lambda^{k} \mu^{l}=0 .
$$


Sufficiency. Assume on the contrary that $\left\{u_{i, j}\right\}$ is an eventually positive solution of Eq. 1. In view of Eq. 1, we have

$$
u_{i+1, j}+u_{i, j+1}=p u_{i, j}-q u_{i+k, j+l}
$$

Since $p \leq 0$ and $q \geq 0$, we can see that the left side of Eq. 3 is strictly greater than 0 and the right sight of Eq. 3 is less than or equal to 0 . This is a contradiction.

Necessity. Assume on the contrary that $p \leq 0$ and $q \geq 0$ do not hold. We only need to consider the following three cases.

Case 1) $p>0$ and $q<0$. Letting $\mu=p+\delta$ in Eq. 2, we obtain

$$
\phi(p, q, \lambda)=q(p+\delta)^{l} \lambda^{k}+\lambda+\delta=0
$$

When $k=1$, it follows from Eq. 4 that $\lambda=-\delta /\left(1+q(p+\delta)^{l}\right)$. Choosing $\delta^{*}>\max \{0, \sqrt[l]{-1 / q}-p\}$, we have $\lambda^{*}=-\delta^{*} /\left(1+q\left(p+\delta^{*}\right)^{l}\right)>0$. It follows from $p>0$ and $\delta^{*}>0$ that $\mu^{*}=p+\delta^{*}>0$. Hence, $\left(\lambda^{*}, \mu^{*}\right)$ is a positive root of Eq. 2. When $k>1$, choosing $\delta=\delta^{*}>0$ in Eq. 4, we have $\lim _{\lambda \rightarrow 0^{+}} \phi(p, q, \lambda)=\delta^{*}>0$ and $\lim _{\lambda \rightarrow+\infty} \phi(p, q, \lambda)=-\infty$. Since Eq. 4 is continuous on $(0,+\infty)$, there exists $\lambda^{*} \in(0,+\infty)$ satisfying Eq. 4 . Notice that $\mu^{*}=p+\delta^{*}>0$ for $p>0$ and $\delta^{*}>0$. Therefore, $\left(\lambda^{*}, \mu^{*}\right)$ is a positive root of Eq. 2 .

Case 2) $p>0$ and $q \geq 0$. Letting $\mu=\lambda$ in Eq. 2, we obtain

$$
\phi(p, q, \lambda)=q \lambda^{k+l}+2 \lambda-p=0
$$

From Eq. 5, we have $\lim _{\lambda \rightarrow 0^{+}} \phi(p, q, \lambda)=-p<0, \lim _{\lambda \rightarrow+\infty} \phi(p, q, \lambda)=+\infty$. Since Eq. 5 is continuous on $(0,+\infty)$, there exists $\lambda^{*} \in(0,+\infty)$ satisfying Eq. 5 . Notice that $\mu^{*}=\lambda^{*}>0$. Therefore, $\left(\lambda^{*}, \mu^{*}\right)$ is a positive root of Eq. 2 .

Case 3) $p \leq 0$ and $q<0$. Letting $\mu=\lambda$ in Eq. 2, we have Eq. 5.

When $p=0$, it follows from Eq. 5 that $\lambda^{*}=k+l-\sqrt[1]{-2 / q}>0$. Notice that $\mu^{*}=\lambda^{*}>0$. Hence, $\left(\lambda^{*}, \mu^{*}\right)$ is a positive root of Eq. 2. When $p<0$, in view of Eq. 5, we have $\lim _{\lambda \rightarrow 0^{+}} \phi(p, q, \lambda)=-p>0$ and $\lim _{\lambda \rightarrow+\infty} \phi(p, q, \lambda)=-\infty$. Since Eq. 5 is continuous on $(0,+\infty)$, there exists $\lambda^{*} \in(0,+\infty)$ satisfying Eq. 5. Notice that $\mu^{*}=\lambda^{*}>0$. Therefore, $\left(\lambda^{*}, \mu^{*}\right)$ is a positive root of Eq. 2.

In all the cases above, Eq. 2 always has positive solutions. This contradicts Lemma 1. The proof is thus completed.

Theorem 2. Assume that $k>1$ and $l=0$ (or $k=0$ and $l>1$ ). Then every solution of Eq. 1 oscillates if and only if $p \leq 0$ and $q \geq 0$.

Proof. When $k>1$ and $l=0$, then the characteristic equation of Eq. 1 is

$$
\phi(p, q, \lambda, \mu)=\lambda+\mu-p+q \lambda^{k}=0 .
$$

Sufficiency. Suppose to the contrary that $\left\{u_{i, j}\right\}$ is an eventually positive solution of Eq. 1. Then from Eq. 1, we have

$$
u_{i+1, j}+u_{i, j+1}=p u_{i, j}-q u_{i+k, j} .
$$

Since $p \leq 0$ and $q \geq 0$, we can see that the left side of Eq. 7 is strictly greater than 0 and the right sight of Eq. 7 is less than or equal to 0 . This is a contradiction. 
Necessity. Suppose that every solution of Eq. 1 is oscillatory. From Lemma 1, one can obtain that Eq. 6 has no positive roots. Substituting $\mu=c \lambda$ into Eq. 6 , we have $c=\left(-q \lambda^{k}-\lambda+p\right) / \lambda$. It is obvious that Eq. 6 has no positive roots if and only if there does not exist $c \in(0,+\infty)$ such that $c=\left(-q \lambda^{k}-\lambda+p\right) / \lambda$ for any $\lambda \in(0,+\infty)$. That is, for any $\lambda \in(0,+\infty), q \lambda^{k}+\lambda-p \geq 0$ always holds. Let $f(\lambda)=q \lambda^{k}+\lambda-p$. Then, we have $f^{\prime}(\lambda)=k q \lambda^{k-1}+1$.

i) When $q \geq 0$, notice that $f^{\prime}(\lambda)>0$ for $\lambda>0$. Therefore, $f(\lambda)$ is strictly increasing on $(0,+\infty)$. For any $\lambda \in(0,+\infty)$, to ensure $f(\lambda) \geq 0$, it only needs to satisfy the relation $f(\lambda) \geq f(0)=-p \geq 0$, that is, $p \leq 0$.

ii) When $q<0$, since $f(\lambda)$ is continuous on $(0,+\infty)$ and $\lim _{\lambda \rightarrow+\infty} f(\lambda)=-\infty$, it is obvious that $f(\lambda) \geq 0$ does not always hold for any $\lambda \in(0,+\infty)$.

Combining i) and ii) implies that necessity is true. The proof is thus completed. The proof for the case $k=0$ and $l>1$ is similar to the case for $k>1$ and $l=0$ and is not repeated here.

Theorem 3. Assume that $k=1$ and $l=0$ (or $k=0$ and $l=1$ ). Then every solution of Eq. 1 oscillates if and only if $p \leq 0$ and $q \geq-1$.

Proof. When $k=1$ and $l=0$, the characteristic equation of Eq. 1 is

$$
(1+q) \lambda+\mu-p=0 .
$$

It is clear that Eq. 8 does not have any positive roots if and only if $p \leq 0$ and $q \geq-1$. Hence, Lemma 1 implies the statement of this theorem. This completes the proof. The proof for the case $k=0$ and $l=1$ is similar to the case for $k=1$ and $l=0$ and is not repeated here.

\section{Illustrative examples}

Example 1. Consider the advanced 2-D discrete system

$$
u_{m+1, n}+u_{m, n+1}+1.05 u_{m, n}+u_{m+1, n+1}=0 .
$$

From Eq. 9, we have $k=1, l=1, p=-1.05, q=1$. Since $p=-1.05<0$ and $q=1>0$, by Theorem 1, every solution of Eq. 9 is oscillatory. The oscillatory behavior of Eq. 9 is demonstrated by Fig. 1 .

Example 2. Consider the advanced 2-D discrete system

$$
u_{m+1, n}+u_{m, n+1}+0.6 u_{m, n}+u_{m+2, n+1}=0 .
$$

From Eq. 10, we have $k=2, l=0, p=-0.6, q=1$. Since $p=-0.6<0$ and $q=1>0$, by Theorem 2, every solution of Eq. 10 is oscillatory. The oscillatory behavior of Eq. 10 is demonstrated by Fig. 2 .

Example 3. Consider the advanced 2-D discrete system

$$
u_{m+1, n}+1.02 u_{m, n+1}+0.1 u_{m, n}=0
$$

From Eq. 11, we have $k=0, l=1, p=-0.1,1+q=1.02$. Since $p=-0.1<0$ and $1+q=1.02>0$, in view of Theorem 3, every solution of Eq. 11 is oscillatory. The oscillatory behavior of Eq. 11 is demonstrated by Fig. 3. 


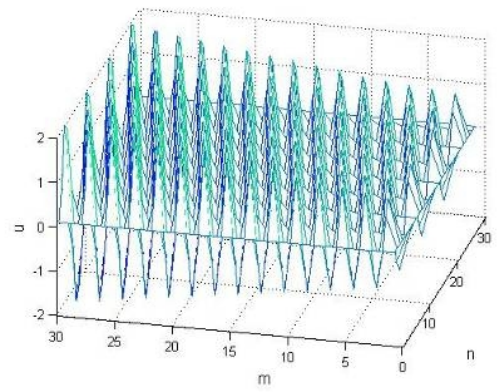

Fig. 1 Oscillatory behavior of Eq. 9

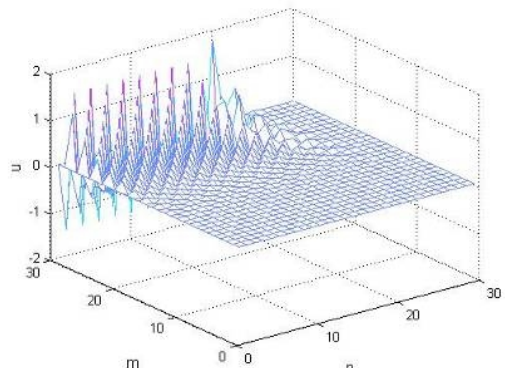

Fig. 2 Oscillatory behavior of Eq. 10

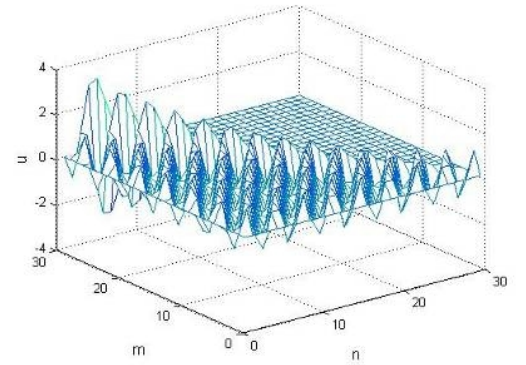

Fig. 3 Oscillatory behavior of Eq. 11

\section{Conclusions}

In this paper, we derived effective criteria to determine oscillations of an advanced 2-D discrete system. Oscillation criteria for advanced 2-D discrete systems are different from delay 2-D discrete systems. Numerical examples are given to illustrate the results presented in this paper.

\section{Acknowledgements}

The research work was supported by Natural Science Foundation of Shandong Province under Grant No. ZR2014FL015 and Natural Science Foundation of Shandong Province under Grant No. ZR2014AP011.

\section{References}

[1] B.E. Shi, L.O. Chua: IEEE Trans. Circuits Syst. I vol. 39 (1992), p. 531

[2] R.J. LeVeque: Numerical Methods for Conservation Laws (Birkhauser-Verlag, GER 1990).

[3] S.S. Cheng: Partial Difference Equations, vol. 3 of Advances in Discrete Mathematics and Applications (Taylor \& Francis, UK 2003).

[4] B.G. Zhang, Y. Zhou: Qualitative Analysis of Delay Partial Difference Equations (Hindawi Publishing Corporation, USA 2007).

[5] B.G. Zhang, Q.J. Xing: J. Math. Anal. Appl. vol. 329 (2007), p. 567

[6] B.G. Zhang, R.P. Agarwal: Comput. Math. Appl. vol. 45 (2003), p.1253

[7] R.P. Agarwal, Y. Zhou: Math. Comput. Model. vol. 31 (2000), p. 17

[8] B.G. Zhang, J.S. Yu: Comput. Math. Appl. Vol. 35 (1998), p.111

[9] P.J.Y. Wong, R.P. Agarwal: Comput. Math. Appl. vol. 32 (1996), p. 57

[10] B.G. Zhang, S.T. Liu: Discrete Dynamics in Nature and Society vol. 1 (1998), p. 265

[11] J.F. Cheng, Y.M. Chu: Bull. Inst. Math. Acad. Sinica vol. 1 (2006), p. 507 